PROCEEDINGS OF THE

AMERICAN MATHEMATICAL SOCIETY

Volume 127, Number 7, Pages 2029-2033

S 0002-9939(99)05147-3

Article electronically published on February 26, 1999

\title{
A SHARP EXPONENTIAL INEQUALITY FOR LORENTZ-SOBOLEV SPACES ON BOUNDED DOMAINS
}

\author{
STEVE HUDSON AND MARK LECKBAND
}

(Communicated by Christopher D. Sogge)

\begin{abstract}
This paper generalizes an inequality of Moser from the case that $\nabla u$ is in the Lebesgue space $L^{n}$ to certain subspaces, namely the Lorentz spaces $L^{n, q}$, where $1<q \leq n$. The conclusion is that $\exp \left(\alpha u^{p}\right)$ is integrable, where $1 / p+1 / q=1$. This is a higher degree of integrability than in the Moser inequality when $q<n$. A formula for $\alpha$ is given and it is also shown that no larger value of $\alpha$ works.
\end{abstract}

For $n \geq 2$, let $D$ be a bounded domain in $R^{n}$ and let $W^{n}(D)$ be the Sobolev space of functions defined as the completion of the space of $C^{\infty}$ functions compactly supported in $D$ whose gradient is in $L^{n}(D)$. A well known result of J. Moser [7] is that, for functions $u$ in the unit ball of $W^{n}(D)$, there is a sharp constant $\alpha=\alpha_{n}=n\left(\sigma_{n-1}\right)^{1 /(n-1)}$, where $\sigma_{n-1}$ is the $n-1$ dimensional surface area of the unit sphere, such that

$$
\int_{D} \exp \left\{\alpha u^{n /(n-1)}\right\} \leq A(n) m(D), \quad A(n) \text { is independent of } u .
$$

We define a Lorentz-Sobolev space $W^{n, q}(D)$ using Lorentz norms and prove a similar sharp exponential inequality. When $q=n, W^{n}(D)=W^{n, n}(D)$.

For real valued functions $f$ on $R^{n}$, let $f^{*}$ be the nonincreasing rearrangement of $f$ defined as $f^{*}(t)=\inf \{s: m\{|f|>s\} \leq t\}$. We define $f^{\#}(x)$ to be the spherically symmetric nondecreasing rearrangement of $f$ defined as $f^{\#}(x)=f^{*}\left(\sigma_{n-1}|x|^{n} / n\right)$.

The Lorentz $L(n, q)$ norm is defined as

$$
\|f\|_{n, q}=\left[(q / n) \int_{0}^{\infty}\left[f^{*}(t) t^{1 / n}\right]^{q} d t / t\right]^{1 / q} .
$$

The constant $(q / n)$ ensures that $\left\|\chi_{E}\right\|_{n, q}=(m(E))^{1 / n}$. For $1<q \leq n$, it is shown in [1] that $L(n, q)$ is an actual norm.

Definition. Define $W^{n, q}(D), 1<q \leq n$, as the completion of the space of functions $u$ in $C^{\infty}$ compactly supported in $D$ satisfying

$$
\|\nabla u\|_{n, q}<\infty .
$$

Received by the editors September 16, 1997.

1991 Mathematics Subject Classification. Primary 46E35; Secondary 46E30.

Key words and phrases. Sobolev imbedding theorem, Moser's inequality, Lorentz space.

(C)1999 American Mathematical Society 
Theorem 1. Let $D$ be a bounded domain in $R^{n}$. For functions $u$ in $W^{n, q}(D)$ such that $\|\nabla u\|_{n, q} \leq 1,1<q \leq n$, there is a sharp constant

$$
\alpha_{n, q}=q^{1 /(q-1)} n^{1-p / n}\left(\sigma_{n-1}\right)^{p / n}
$$

such that for $p=q /(q-1)$,

$$
\int_{D} \exp \left\{\alpha_{n, q}|u(x)|^{p}\right\} d x \leq A(q) m(D) .
$$

This theorem generalizes the above result of [7] and fits nicely with the results of [3] and [4]. Consider a result of Fusco, Lions, and Sbordone [4]. They have shown that the Zygmund-Sobolev space, defined as the set of functions $u$ for which $\nabla u$ belongs to the Zygmund space $L^{n}\left(\log ^{-r}(L)\right)(D), r>0$, can be continuously imbedded into the Orlicz space $L_{\exp , n /(n-1+r)}(D)$, that is, the linear hull of the set of all functions $f$ such that

$$
\int_{D} \exp \left\{|f(x)|^{n /(n-1+r)}\right\} d x<\infty
$$

Since the exponent $p$ for $1<q \leq n$ satisfies $p \geq n /(n-1)>n /(n-1+r)$, Theorem 1 makes a stronger conclusion with a stronger hypothesis. However, simple examples show the theorem cannot be extended to the range $q>n$.

It is not known if Theorem 1 has extremals. The authors show in [5] that the closely related Theorem A below has extremals for all $q>1$.

\section{Proof of ThEOREM 1}

Letting $t=|x|^{n} \sigma_{n-1} / n$, we may rewrite the $L(n, q)$ norm of $f$ in terms of $f^{\#}$ as

$$
\|f\|_{n, q}=\left[\left(n / \sigma_{n-1}\right)^{1-q / n}(q / n) \int\left(f^{\#}(x)\right)^{q}|x|^{q-n} d x\right]^{1 / q} .
$$

Our proof is based on the following one dimensional inequality.

Theorem A (Jodeit [6], Moser [7]). Let $1<q<\infty, 1 / p+1 / q=1$. Let $\omega$ be a function in $C^{1}[0, \infty)$ such that $\omega(0)=0$ and $\int_{0}^{\infty}\left|\omega^{\prime}(t)\right|^{q} d t \leq 1$. Then $\sup _{\omega} \int_{0}^{\infty} \exp \left\{\omega^{p}(t)-t\right\} d t=A(q)<\infty$.

We translate the statement of Theorem A to Theorem 1 by identifying $|x| / R=$ $e^{-t / n}$ and $\omega(t)=\alpha^{1 / p} u^{\#}(x)$, where $u^{\#}$ is the symmetrical rearrangement of a function $u$ in $C^{1}(D)$ vanishing at the boundary of $D$ and $R$ is defined by $m(D)=$ $m\left(B_{R}(0)\right)$. We may assume $R=1$. The constant $\alpha$ is determined after we carry out the change of variables. Observe $d|x| / d t=-|x| / n$ and

$$
\omega^{\prime}(t)=\left(\alpha^{1 / p}|x| / n\right)|\nabla u|^{\#}(x), \quad x \text { in } B_{1}(0) .
$$

So the conclusion of Theorem A is that the (Moser) functional

$$
F\left(u^{\#}\right)=F(u)=\left(n / \sigma^{n-1}\right) \int \exp \left\{\alpha u^{p}(x)\right\} d x
$$

is bounded given that

$$
G^{q}\left(u^{\#}\right)=\alpha^{q-1}\left(n^{1-q} / \sigma_{n-1}\right) \int\left(|\nabla u|^{\#}(x)\right)^{q}|x|^{q-n} d x \leq 1 .
$$


We define

$$
\alpha=\alpha_{n, q}=q^{1 /(q-1)}\left(\sigma_{n-1}\right)^{p / n} n^{1-p / n} .
$$

This ensures that (4) resembles (1).

Lemma 1. Let $u \geq 0$ be continuously differentiable on D, compactly supported in $D$, and all of the nonzero level sets have $n$-dim measure zero. For $n \geq 2,1<q \leq n$, we have

$$
G\left(u^{\#}\right) \leq\left[\|\nabla u\|_{n, q}\right] .
$$

Proof. Let $c_{n, q}$ be the constant of (1).

$$
\begin{aligned}
G^{q}\left(u^{\#}\right) & =c_{n, q} \int\left|\nabla u^{\#}(x)\right|^{q-1} \cdot|x|^{q-n}|\nabla u \#(x)| d x \\
& =c_{n, q} \int_{0}^{\infty} \int_{\left(u^{\#}\right)^{-1}(t)} f(x) d H^{n-1} x d t,
\end{aligned}
$$

where $f(x)=\left|\nabla u^{\#}(x)\right|^{q-1} \cdot|x|^{q-n}$ is a radial function. Let $\Phi_{1}$ and $\Phi_{2}$ be defined by the equations

$$
\begin{aligned}
& \Phi_{1}\left(u^{\#}(x)\right)=f(x), \\
& \Phi_{2}\left(u^{\#}(x)\right)=|x|^{(n-q) / q} .
\end{aligned}
$$

Then, using the isoperimetric inequality,

$$
\begin{aligned}
G^{q}\left(u^{\#}\right) c_{n, q} & =\int_{0}^{\infty} \int_{\left(u^{\#}\right)^{-1}(t)} \Phi_{1}\left(u^{\#}(x)\right) d H^{n-1} x d t, \\
& \leq \int_{0}^{\infty} \int_{(u)^{-1}(t)} \Phi_{1}(u(x)) d H^{n-1} x d t, \\
& =\int \Phi_{1}(u(x)) \Phi_{2}(u(x)) / \Phi_{2}(u(x))|\nabla u(x)| d x, \\
& \leq\left[\int\left(\Phi_{1}(u(x)) \Phi_{2}(u(x))\right)^{p} d x\right]^{1 / p}\left[\int\left|\nabla u(x) / \Phi_{2}(u(x))\right|^{q} d x\right]^{1 / q} .
\end{aligned}
$$

Using the equimeasurability of $u$ and $u^{\#}$, the first integral on the right is simply $\left[G^{q}\left(u^{\#}\right) / c_{n, q}\right]^{1 / p}$. The second integral is bounded by

$$
\left[\int_{0}^{\infty}\left(|\nabla u|^{*}(t)\right)^{q}\left[\left(1 / \Phi_{2}(u)\right)^{*}(t)\right]^{q} d t\right]^{1 / q} .
$$

We compute using $\left(1 / \Phi_{2}(u)\right)^{*}(t)=\inf \left\{s: m\left\{x: 1 / \Phi_{2}(u(x))>s\right\} \leq t\right\}$, the equimeasurability of $u$ and $u^{\#}$, and the definition of $\Phi_{2}$ that

$$
\left(1 / \Phi_{2}(u)\right)^{*}(t)=\left[\sigma_{n-1} /(t n)\right]^{(n-q) /(q n)} .
$$

So (7) is equal to

$$
\left[\left(\sigma_{n-1} / n\right)^{(n-q) / n} \int\left(|\nabla u|^{*}(t) t^{1 / n}\right)^{q} d t / t\right]^{1 / q}=c_{n, q}^{-1 / q}\|\nabla u\|_{n, q} .
$$


So, by (6) and (8),

$$
G^{q}\left(u^{\#}\right) \leq G^{q / p}\left(u^{\#}\right)\|\nabla u\|_{n, q} .
$$

This proves Lemma 1 which implies Theorem 1 for a dense class of functions. An application of Fatou's Lemma completes the proof of Theorem 1.

\section{The CONSTANT $\alpha^{n, q}$ OF TheOREM 1 IS SharP}

In [7], Moser shows with a simple example that Theorem A is sharp. Unfortunately, the sharpness of Theorem A implies the sharpness of Theorem 1 only for $q=n$. However the computations below allow us to modify Moser's example and establish the sharpness of $\alpha_{n, q}$.

Let $D$ be $B_{1}(0)$. Let $a>1$ and $0<\delta<1$. We will choose $a$ and $\delta$ later. Define $\omega(0)=0, \omega^{\prime}(t)=\delta a^{-1 / q}$ for $0 \leq t \leq a$, and 0 otherwise. Then we use equation (2), with $\alpha$ replaced by $\alpha_{n, q}$, to define a radial Lorentz-Sobolev function $u$ whose gradient is supported in the annulus of radii $e^{-a / n}$ and 1 centered at $x_{0}$. We claim that

$$
\left[\|\nabla u\|_{n, q}\right]^{q} \leq \delta^{q}[1+n /(q a)] .
$$

Assuming the claim, we now construct an example to show that $\alpha_{n, q}$ is maximal. Let $\alpha_{2}>\alpha_{n, q}$. Then for $\beta=\alpha_{2} / \alpha_{n, q}>1, c=\sigma_{n-1} / n$

$$
\begin{aligned}
\int_{B} \exp \left\{\alpha_{2} u^{p}\right\} d x & =c \int_{0}^{\infty} \exp \left\{\beta \omega^{p}(t)-t\right\} d t \\
& \geq c \int_{a}^{\infty} \exp \left\{\beta \delta^{p} a-t\right\} d t \\
& =c \exp \left\{a\left(\beta \delta^{p}-1\right)\right\} .
\end{aligned}
$$

Now choose $\delta<1$ so that $\delta^{p}>1 / \beta$. Then for all large enough $a,\|\nabla u\|_{n, q} \leq 1$, yet $\exp \left\{a\left(\beta \delta^{p}-1\right)\right\}$ is unbounded.

To establish our claim, be begin by computing $(|\nabla u|)^{*}$. Let $C=n \delta / \alpha_{n, q}^{1 / p}$. Observe for $e^{-a / n} \leq|x| \leq 1$, that $\max |\nabla u|=C a^{-1 / q} e^{a / n}$ and $\min |\nabla u|=$ $C a^{-1 / q}$. So, for $C a^{-1 / q} \leq s \leq C a^{-1 / q} e^{a / n}$, we have $t=m\{|\nabla u|>s\}=$ $\left(\sigma_{n-1} / n\right)\left[\left(C a^{-1 / q} / s\right)^{n}-e^{-a}\right]$. Therefore, solving for $s$ gives us

$$
(|\nabla u|)^{*}(t)= \begin{cases}\frac{C a^{-1 / q}}{\left[\left(n / \sigma_{n-1}\right) t+e^{-a}\right]^{1 / n}}, & 0 \leq t \leq \sigma_{n-1}\left[1-e^{-a}\right] / n, \\ 0, & \text { otherwise. }\end{cases}
$$

We compute

$$
\begin{aligned}
{\left[\|\nabla u\|_{n, q}\right]^{q} } & =C^{q} q /(\text { an }) \int_{0}^{\sigma_{n-1}\left[1-e^{-a}\right] / n} \frac{t^{q / n}}{\left[\left(n / \sigma_{n-1}\right) t+e^{-a}\right]^{q / n}} \frac{d t}{t} \\
& =\delta^{q} / a \int_{0}^{\left(1-e^{-a}\right)} \frac{s^{q / n}}{\left[s+e^{-a}\right]^{q / n}} \frac{d s}{s} \\
& =\delta^{q} / a \int_{0}^{e^{a}-1}[w /(w+1)]^{q / n} \frac{d w}{w} .
\end{aligned}
$$

By considering the integration over $[0,1]$ and $\left[1, e^{a}-1\right]$ separately, the above is less than $\left(\delta^{q} / a\right)[n / q+a]$. 


\section{REFERENCES}

[1] C. Bennett and R. Sharpley, Interpolation of operators, Vol. 129, Pure and Applied Math., Acad. Press, Inc. MR 89e:46001

[2] L. Carleson and S. Y. A. Chang, On the existence of an extremal function for an inequality of J. Moser, Bull. Sc. Math., $2^{e}$ série, 110 (1986), 113-127. MR 88f:46070

[3] D. E. Edmunds and H. Triebel, Logarithmic Sobolev spaces and their applications to spectral theory, Proc. London Math. Soc., 71 (1995), No. 3, 333-371. MR 96f:46061

[4] N. Fusco, P. L. Lions and C. Sbordone, Some remarks on Sobolev imbeddings in borderline cases, Preprint No. 25, Universita degli Studia di Napoli, "Feder II", 1993. MR 94e:49013

[5] S. Hudson and M. Leckband, Extremals for a Moser-Jodeit exponential inequality, preprint.

[6] M. Jodeit, An inequallity for the indefinite integral of a function in $L^{q}$, Studia Math., 44 (1972), 545-554. MR 49:5805

[7] J. Moser, A sharp form of an inequality by N. Trudinger, Ind. Univ. Math. J., 23 (1971), 1077-1092. MR 46:662

[8] N. S. Trudinger, On imbeddings into Orlicz spaces and some applications, J. Math. Mech., 17 (1967), 473-484. MR 35:7121

Department of Mathematics, Florida International University, University Park, MiAMi, FLORIDA 33199

E-mail address: hudsons@fiu.edu

E-mail address: leckband@fiu.edu 